\title{
Asian Longhorned Tick Haemaphysalis longicornis Neumann (Arachnida: Acari: Ixodidae) ${ }^{1}$
}

\author{
Yuexun Tian and Phillip E. Kaufman ${ }^{2}$
}

\section{Introduction}

The Asian longhorned tick (Figure 1), Haemaphysalis longicornis Neumann, also known as the longhorned tick, bush tick (Australia), and cattle tick (New Zealand), is a three-host tick native to East Asia (Rainey et al. 2018). This tick has been categorized as an invasive species in Australia, New Zealand, and several Pacific Islands (Sleeman 2018). In the US, an Asian longhorned tick infestation was recorded in New Jersey during the fall of 2017. This tick is somewhat unique in that females are capable of parthenogenesis, meaning that females can produce offspring without having mated with males (Burtis 2018). The Asian longhorned tick feeds on a wide range of hosts including birds and mammals, including humans (Rainey et al. 2018). This tick is considered a medical and veterinary vector due to its ability to transmit pathogens that cause disease in humans and other animals.

\section{Distribution}

The Asian longhorned tick is native to Southeast Asia, including: China, Japan, Korea, Vietnam, Thailand, and the former Union of Soviet Socialist Republics (USSR). This species has established invasive populations in eastern Australia (southwestern Queensland, coastal New South Wales, and Victoria), New Zealand, and several Pacific Islands (New Caledonia, Fiji, Western Samoa, Tonga, and Vanutu) (Heath et al. 2011).

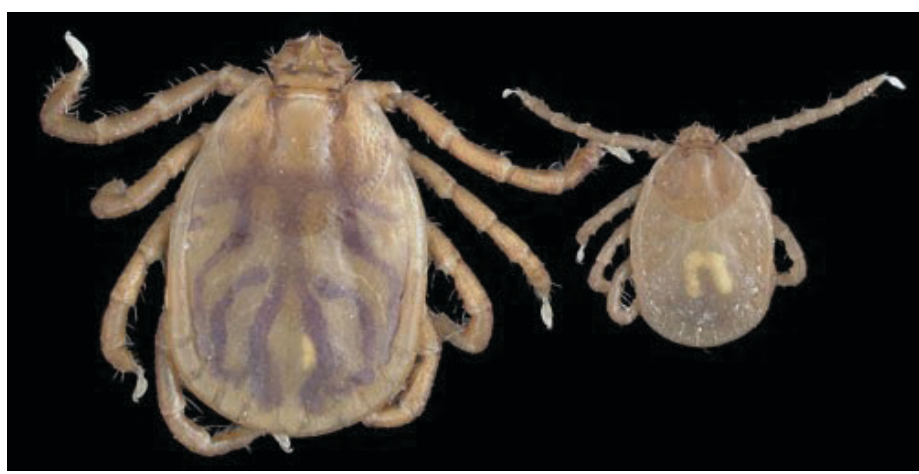

Figure 1. The female (left) and nymph (right) of the Asian longhorned tick, Haemaphysalis longicornis Neumann.

Credits: Lyle Buss, UF/IFAS

In August 2017, a tick from an Icelandic sheep was brought to the Hunterdon County Health Department in New Jersey. The tick was identified as an Asian longhorned tick, Haemaphysalis longicornis, in November 2017 by the National Veterinary Services Laboratory (Rainey et al. 2018). In early October, a large number of ticks were found both on sheep and in paddocks in Hunterdon County, New Jersey (Rainey et al. 2018). At this time, the tick was reported as a new introduction in the US. However, following this initial report, a search of archived tick specimens at the National Veterinary Services Laboratory found that Haemaphysalis longicornis was collected as early as 2010 in West Virginia. These earlier specimens were misidentified initially as a native rabbit tick, Haemaphysalis leporispalustris Packard. Another early specimen was confirmed from a 2013 collection in New Jersey. Since these initial collections,

1. This document is EENY-739, one of a series of the Entomology and Nematology Department, UF/IFAS Extension. Original publication date August 2019. Visit the EDIS website at https://edis.ifas.ufl.edu for the currently supported version of this publication. This document is also available on the Featured Creatures website at http://entomology.ifas.ufl.edu/creatures.

2. Yuexun Tian, Florida Medical Entomology Laboratory; and Phillip E. Kaufman, professor, Entomology and Nematology Department; UF/IFAS Extension, Gainesville, FL 32611.

The Institute of Food and Agricultural Sciences (IFAS) is an Equal Opportunity Institution authorized to provide research, educational information and other services

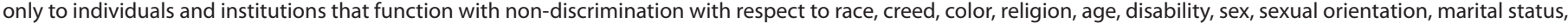

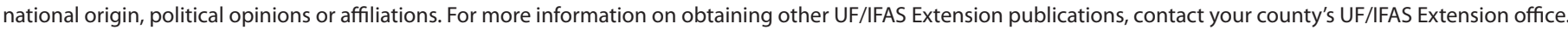
U.S. Department of Agriculture, UF/IFAS Extension Service, University of Florida, IFAS, Florida A \& M University Cooperative Extension Program, and Boards of County Commissioners Cooperating. Nick T. Place, dean for UF/IFAS Extension. 
the Asian longhorned tick has been found in numerous states, suggesting that the ticks are either expanding their distribution or have gone unnoticed in their current distribution. To date (6 June 2019), infestations have been confirmed in 11 states: Arkansas, Connecticut, Kentucky, Maryland, New Jersey, New York, North Carolina, Pennsylvania, Tennessee, Virginia, and West Virginia (Figure 2).

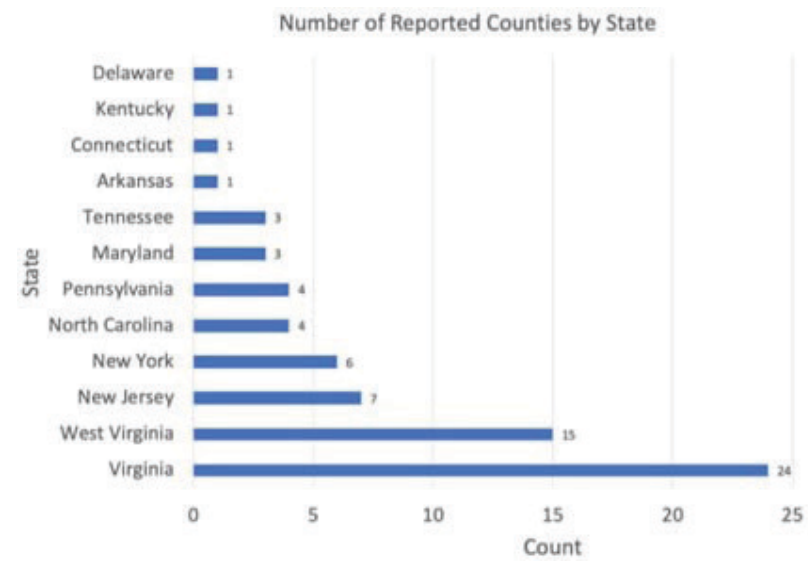

Figure 2. The number of counties in multiple states where positive identifications of Asian longhorned ticks, Haemaphysalis longicornis Neumann, have been made, as of 4 August 2019.

Credits: Graph by Yuexun Tian; Data source: United State Department of Agriculture

According to the predictions from best-fitting models, there is a potential for broad distribution in North America for Asian longhorned ticks, but the southeastern US., the Pacific Northwest, and central and southern Mexico are the three geographic areas with the highest probability for the ticks future distribution (Raghavan et al. 2019). However, this model was developed based on the tick distribution in the native range. Thus, the influence of North American environmental effects on Asian longhorned tick. distribution is not clear.

\section{Description}

Larvae of Asian longhorned tick have three pairs of legs with a body size of approximately 0.58 to $0.62 \mathrm{~mm}$ in length and 0.47 to $0.51 \mathrm{~mm}$ in width (Hoogstraal et al. 1968). The scutum (shield-like feature on back) of the larva is approximately 1.6 times as wide as it is long (Hoogstraal et al. 1968). Both nymphs and adults have four pairs of legs. They can be distinguished by the presence of genital pores on adults and absence on nymphs (Figure 3 ). An unengorged (prior to a blood meal) nymph is about $1.76 \mathrm{~mm}$ in length and $1 \mathrm{~mm}$ in width. The scutum of the nymph is approximately 1.25 times as wide as long, and its outline is broadly rounded (Figure 1, Hoogstraal et al. 1968). Females and males are reddish-yellow in color, but have different body sizes. Females are 2.7 to $3.4 \mathrm{~mm}$ in length and 1.4 to $2 \mathrm{~mm}$ in width (Figure 4 and Figure 5), while the smaller males are approximately $2.51 \mathrm{~mm}$ in length and $1.65 \mathrm{~mm}$ in width. The scutum of females is smaller than that of males, covers only the anterior dorsal surface, and has an angular margin that becomes more obvious during blood feeding (Hoogstraal et al. 1968). The scutum on males covers the entire dorsal surface. For more details of description, see Hoogstraal et al. (1968).

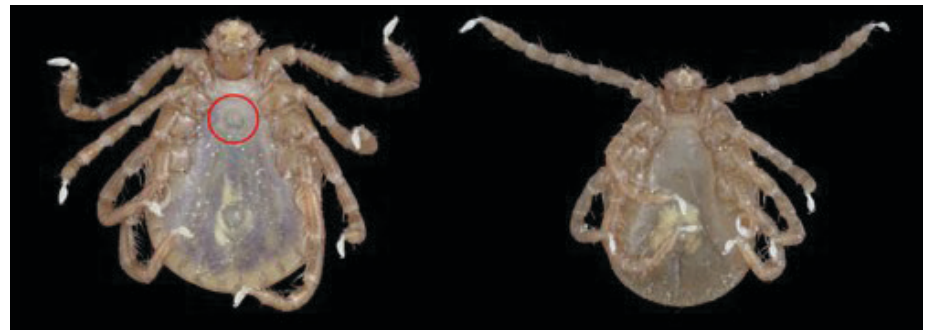

Figure 3. Asian longhorned tick, Haemaphysalis longicornis Neumann, female (left) and nymph (right) on ventral view. Note the genital pore (red circle) on females.

Credits: Lyle Buss, UF/IFAS

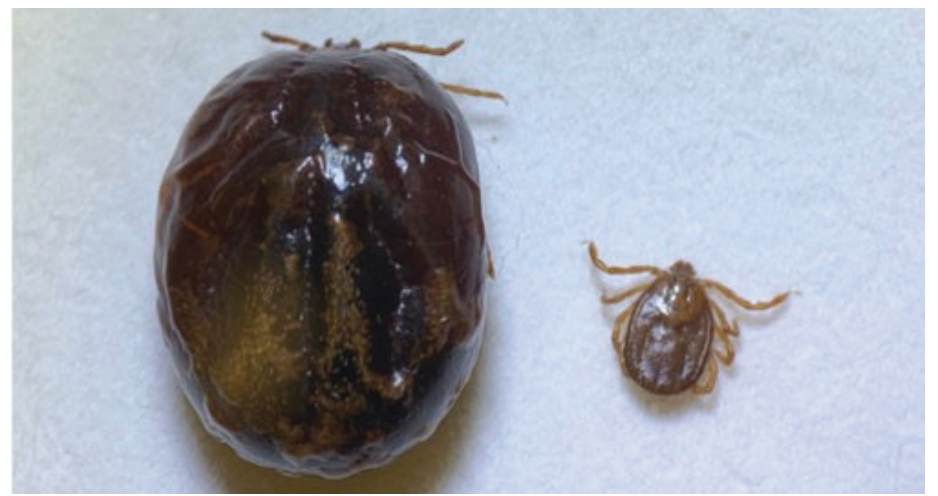

Figure 4. Asian longhorned tick, Haemaphysalis longicornis Neumann, blood fed, engorged (left) and non-blood fed, flat female (right). Credits: Yuexun Tian, UF/IFAS

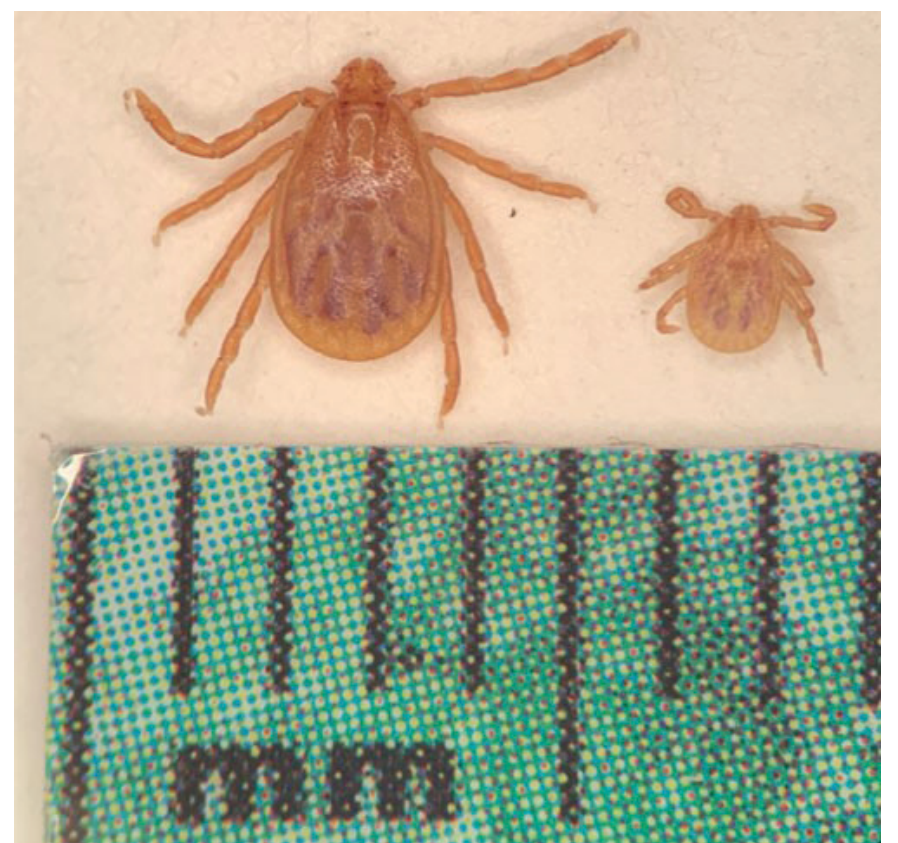

Figure 5. Asian longhorned tick, Haemaphysalis longicornis Neumann, female (left) and nymph (right).

Credits: Yuexun Tian, UF/IFAS 
Haemaphysalis ticks have a similar body size to the blacklegged tick, Ixodes scapularis Say, which is small when compared to the long star tick, Amblyomma americanum (Linnaeus), Haemaphysalis ticks and do not have eyes, but do have festoons (wrinkles or indentations located at the rear edge of the tick). A distinguishing feature of this tick genera is that the second segment of the palpi (the parts on both sides of the mouthparts) extend laterally (to the side) beyond the rectangular basis capituli (area between the mouthparts and main body) (Figure 7, Burtis 2018).

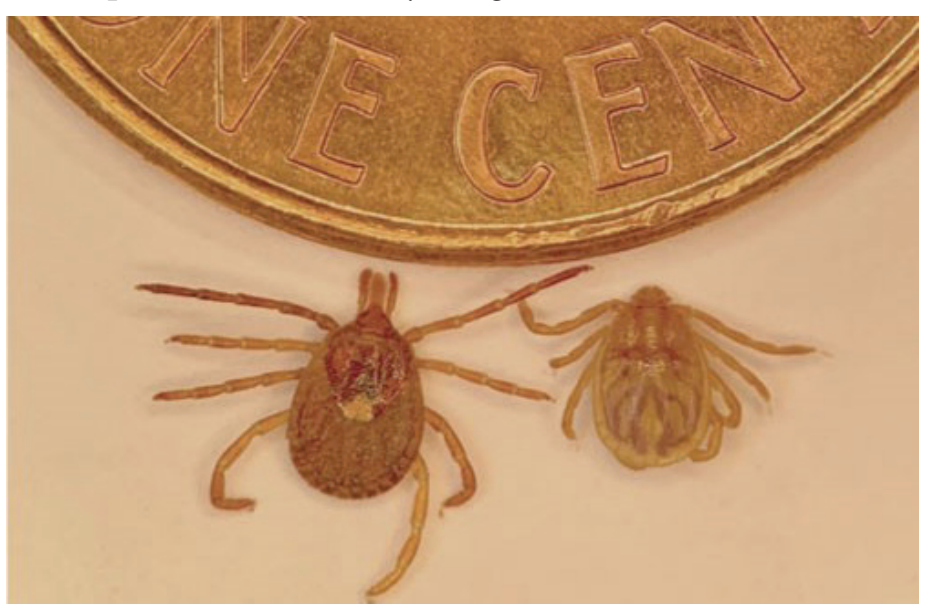

Figure 6. The adult stage of the lone star tick (left), Amblyomma americanum Linnaeus, and female Asian longhorned tick (right), Haemaphysalis longicornis Neumann, compared with a one cent coin. Note the short angular mouthparts on the Asian longhorned tick as compared to long and narrow mouthparts on the long star tick. Credits: Yuexun Tian, UF/IFAS

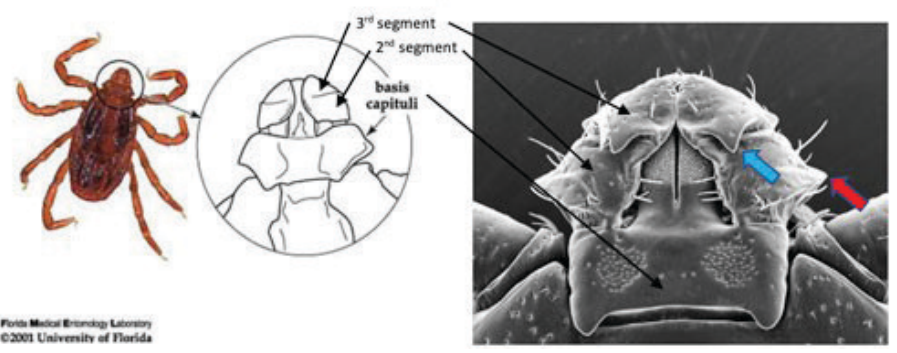

Figure 7. Mouthparts of the Asian longhorned tick (right), Haemaphysalis longicornis Neumann, and the brown dog tick (left), Rhipicephalus sanguineus Latreille, on dorsal side. Note the angular feature of the second segment of palps (red arrow) and the spur on the third segment of palps (blue arrow) on the Asian longhorned tick. The angular feature on the brown dog tick is not on the mouthparts and there is not a spur on the third palpal segment.

Credits: Andrea M. Egizi (right), Monmouth County Mosquito Control Division, NJ; and James Newman (left), UF/IFAS

The rabbit tick (Haemaphysalis leporispalustris) and bird tick (Haemaphysalis chordeilis) are two Haemaphysalis species native to the US and are distributed across the country (Burtis 2018). Haemaphysalis longicornis can be distinguished from the rabbit tick by the presence of the dorsal spur on the third palpi segment (Figure 7). Asian longhorned ticks are difficult to identify by non-experts.
Given the importance of this new invasive species, if suspected Asian longhorned ticks are found in Florida, a sample should be sent to Dr. Phillip Kaufman at the UF Entomology and Nematology Department's Veterinary Laboratory (contact pkaufman@ufl.edu for sample submission information) for accurate identification, and to allow proper tracking of this important tick.

\section{Life Cycle and Biology}

The Asian longhorned tick is a three-host tick; meaning that after taking a blood meal, each active stage (larva, nymph, and adult) will leave the host to digest the blood meal, and develop and molt into the next stage, or if an adult female will lay eggs and die. The engorged (fully blood-fed) female can produce up to 2,000 eggs over 2 to 3 weeks (Machtinger and Skvarla 2018). The larvae hatch about 25 days after oviposition when held at $25^{\circ} \mathrm{C}$ (Yano et al. 1987). These newly-hatched larvae immediately search for a host. After locating and attaching to a host, they feed for 3 to 9 days (Heath 2016). Once larvae are engorged, they drop from the host and digest the blood meal before molting into nymphs, a period that may take up to two weeks (Yano et al. 1987).

Nymphs then locate and attach to a new host, feed for 3 to 8 days, and fall from the second host, where they take about 17 days to digest their blood meal and develop into adults. After finding a suitable host, adults may feed for 7 to 14 days before dropping from this third host. The Asian longhorned tick can compete its life cycle in six months, but typically one generation occurs per year, with most immature ticks entering a dormant state, such as diapause, during winter and other cold periods. The cues for ticks to enter diapause are both abiotic such as temperature, humidity, and photoperiod, and biotic such as nutrition (Heath 2016). For nymphs, photoperiod and temperature are the only cues to induce diapause due to their resistance to dehydration (Heath 2016).

The reproduction of Asian longhorned ticks is unusual among tick species. They can produce offspring by both sexual reproduction (offspring that develop from eggs fertilized by a male) and parthenogenetic reproduction (offspring that develop from unfertilized eggs, from an unmated female). Males are usually uncommon in invasive populations, such as the populations in Australia and New Zealand, and this appears to be the situation in the U.S. populations; however, this remains speculative to date. The ability of female Asian longhorned ticks to produce offspring in the absence of males could be the reason that 
this tick has spread rapidly and reached high abundances in Australia and New Zealand.

The annual pattern of Asian longhorned tick populations is dependent on temperature, humidity, day length, and host availability. Temperature and day length are the major factors determining the time to complete each stage. At temperatures ranging from $12^{\circ} \mathrm{C}$ to $30^{\circ} \mathrm{C}$, it takes 17 to 100 days for larvae to hatch, 11 to 45 days for blood fed larvae to molt into nymphs, and 13 to 63 days for blood fed nymphs to molt into adults (Yano et al. 1987). Generally, the developmental rate increases as temperature increases, but slows as the temperature reaches the upper developmental threshold, which is near $40^{\circ} \mathrm{C}$ (Heath 2016).

Ticks with larger body sizes may need longer periods to feed and develop (the adults feed for a longer time than the larvae). In addition, the feeding period is extended when a larger number of ticks are present on same host (Kang 1981). Dehydration is the most limiting factor for tick survival because the ticks spend approximately $90 \%$ of their life off hosts in potentially desiccating environments (Heath 2016). Unfed Asian longhorned ticks tend to lose water more rapidly than engorged ticks of the same species. However, they have the ability to withstand dehydration. For unfed ticks, nymphs have the greatest resistance to dehydration, followed by larvae and adults. While for engorged individuals, adults have the greatest resistance to dehydration followed by nymphs and larvae (Heath 2016).

\section{Hosts}

The Asian longhorned tick feeds on a wide range of hosts including birds and mammals, with the choice dependent on local host availability (Fonseca et al. 2017). In New Zealand, Asian longhorned ticks prefer to feed on cattle, goats, sheep, and deer, on which heavy infestations can occur (Figure 8). Humans and other animals, such as dogs, horses, cats, and rabbits, have been documented as hosts for Asian longhorned ticks (Fonseca et al. 2017). In the US, immature ticks might feed mainly on white-tailed deer or medium-sized animals according to the sample results in New York (Tufts et al. 2019). To date, there are several confirmed hosts including humans in the US (SCWDS 2019).

About one fourth of US-collected ticks have been obtained from environmental sampling, rather than from a host (SCWDS 2019). This result may be due to Asian longhorned ticks spending $90 \%$ of their lifetime time off host, usually in the soil or at the base of plants (Fonseca et al. 2017). As with many tick species, limited contact with hosts, compared to time off host, may limit the effectiveness of topical livestock pesticide applications (Fonseca et al. 2017).

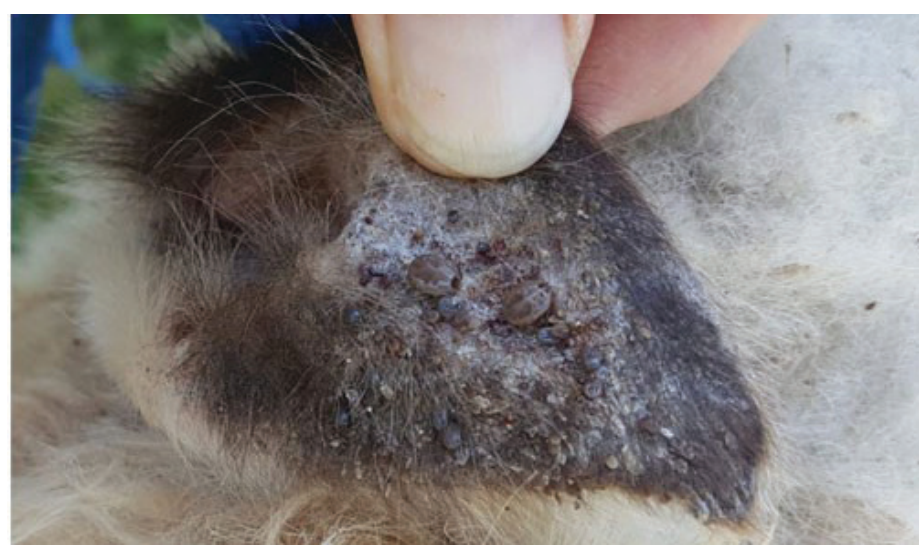

Figure 8. Asian longhorned ticks, Haemaphysalis longicornis Neumann, on a female Icelandic sheep.

Credits: Tadhgh Rainey, Hunterdon County Health Services, NJ

\section{Medical and Veterinary Significance}

The Asian longhorned tick has been demonstrated to be the vector of several pathogens that cause disease in livestock, companion animals, and humans in Asia, such as Babesia gibsoni, Ehrlichia chaffeensis, and Powassan virus. To date, there have been no reports of pathogen infection in Asian longhorned ticks collected in the US; however, it is unknown if this tick will be a competent vector of native pathogens.

Babesia gibsoni is one of the pathogens that causes canine babesiosis. The specific vector of Babesia gibsoni in the US is unknown, but is suspected to be the brown dog tick, Rhipicephalus sanguineus (Boozer and Macintire 2005). Before 1990, Babesia gibsoni infections had been reported only twice in the US (Boozer and Macintire 2005); however, it has been recognized as an important pathogen that affects dogs, especially American pit bull terriers, and it is now diagnosed in many areas of the US.

Ehrlichia chaffeensis is a pathogen that causes human ehrlichiosis, with the white-tailed deer (Odocoileus virginianus Zimmermann) as the principal wildlife reservoir, and the lone star tick, Amblyomma americanum, as the primary vector in the southern, southcentral, and mid-Atlantic regions of the US (Kocan et al. 2000). During 2000 to 2017, the annual number of ehrlichiosis human cases due to Ehrlichia chaffeensis increased from 200 to 1,642 (CDC 2019). Asian longhorned ticks in Korea and China have shown to be infected with Ehrlichia chaffeensis (Kim et al. 2003, Sun et al. 2008), which suggests a potential role for this tick in the US transmission cycle. 
The Asian longhorned tick is a potential vector of Powassan virus, which can infect human and other animals such as woodchucks and snowshoe hares in the US and Canada (Calisher 1994). Powassan is an emerging disease in Canada and the northern US (Cane 2010). The virus usually is transmitted by Ixodes cookei Packard, Ixodes marxi Banks, and Ixodes scapularis Say. From 2008 to 2017, there were 114 Powassan virus cases reported in the US (CDC 2018). Severe fever with thrombocytopenia syndrome virus causes a newly emerging disease that was first recognized in China in 2009 (Luo et al. 2015). This virus is transmitted by Asian longhorned ticks in China, Korea, and Japan, but it has not been found infecting this tick in the US to date.

\section{Management}

The management of ticks can be difficult as ticks spend most of their time off host. Like native tick species, the best management strategy is prevention of tick contact with hosts. Woodlands provide an ideal habitat for most ticks. Removing woody debris from pastures and lawn edges, and maintaining a nine-foot distance between pasture or lawn and wooded edge habitat can reduce the risk of tick contact with humans, companion animals and livestock (Machtinger and Skvarla 2018). Preventing the females from getting a blood meal is critical for effective tick management because of their high reproductive capacity. Thus, livestock and companion animals should be examined regularly, and any attached ticks should be manually removed. Personal tick checks after being outside in tick habitats are the simplest and most effective way to prevent tick infestations. Certain registered repellent products can be applied on skin and clothes (https://www. epa.gov/insect-repellents/find-repellent-right-you), Skvarla and Machtinger 2018). There are some pesticide products available for tick management on animals, and to date (21 May 2019) there is no evidence that Asian longhorned ticks have developed resistance to any of these pesticides (Heath and Levot 2015). Discuss any pesticide treatments with your veterinarian or local UF/IFAS Extension office before applying any treatment and ask for recommendations that fit your needs. Compared with physical check, Extra caution is needed for pesticide handling or application to prevent potential harm on humans and other animals. All pesticides should be used according to the product label.

\section{Selected References}

Boozer L, Macintire D. 2005. "Babesia gibsoni: An emerging pathogen in dogs." Compendium on Continuing Education for the Practicing Veterinarian 27: 33-42.
Burtis J, Egizi A, Occi J, Mader E, Lejeune M, Stafford K, Harrington L. 2018. Intruder alert: Longhorned tick. Northeast Regional Center for Excellence in Vector-Borne Diseases. https://www.acq.osd.mil/eie/afpmb/docs/bulletins/Longhorned_Tick_Fact_Sheet.pdf (Last accessed 21 May 2019).

Calisher CH. 1994. "Medically important arboviruses of the United States and Canada." Clinical Microbiology Review 7: 89-116.

Cane R. 2010. Profile: Haemaphysalis longicornis Neumann, 1901. https://www.smsl.co.nz/site/southernmonitoring/ files/NZB/Ha\%20longicornis\%20Profile.pdf (21 May 2019).

Centers for Disease Control and Prevention (CDC). 2016. Ehrlichiosis: Epidemiology and statistics. https://www.cdc. gov/ehrlichiosis/stats/index.html (21 May 2019)

Centers for Disease Control and Prevention (CDC). 2017. Powassan Virus. https://www.cdc.gov/powassan/index.html (21 May 2019).

Fonseca DM, Egizi A, Occi J. 2017. Review of the biology and ecology of Haemaphysalis longicornis Neumann, 1901. https://fonseca-lab.com/research/global-health-the-tickthat-binds-us-all/ (21 May 2019)

Heath ACG. 2016. "Biology, ecology and distribution of the tick, Haemaphysalis longicornis Neumann (Acari: Ixodidae) in New Zealand." New Zealand Veterinary Journal 64: $10-20$.

Heath ACG, Levot GW. 2015. "Parasiticide resistance in flies, lice and ticks in New Zealand and Australia: Mechanisms, prevalence and prevention." New Zealand Veterinary Journal 63: 199-210.

Heath AC, Occi JL, Robbins RG, Egizi A. 2011. "Checklist of New Zealand ticks (Acari: Ixodidae, Argasidae).” Zootaxa 2995: 55-63.

Hoogstraal H, Roberts FHS, Kohls GM, Tipton VJ. 1968. "Review of Haemaphysalis (Kaiseriana) longicornis Neumann (Resurrected) of Australia, New Zealand, New Caledonia, Fiji, Japan, Korea, and Northeastern China and USSR and its parthenogenetic and bisexual populations (Ixodoidea: Ixodidae)." Journal of Parasitology 54: 1197-1213. 
Kang YB. 1981. "Ecological and physiological properties of the tick, Haemaphysalis longicornis Neumann (Acarina: Ixodidae) with reference to colonization and water relations." Research Report of the Office of Rural Development (Livestock and Veterinary, Korea) 23: 1-47.

Kim CM, Kim MS, Park MS, Park JH, Chae JS. 2003. "Identification of Ehrlichia chaffeensis, Anaplasma phagocytophilum, and A. bovis in Haemaphysalis longicornis and Ixodes persulcatus ticks from Korea." Vector-Borne and Zoonotic Diseases 3: 17-26.

Kocan AA, Levesque GC, Whitworth LC, Murphy GL, Ewing SA, Barker RW. 2000. "Naturally occurring Ehrlichia chaffeensis infection in coyotes from Oklahoma." Emerging Infectious Diseases 6: 477-480.

Luo LM, Zhao L, Wen HL, Zhang ZT, Liu JW, Fang LZ, Xue ZF, Ma DQ, Zhang XS, Ding SJ, Lei XY, Yu XJ. 2015. "Haemaphysalis longicornis ticks as reservoir and vector of severe fever with thrombocytopenia syndrome virus in China." Emerging Infectious Diseases 21: 1770-1776.

Machtinger E, Skvarla MJ. 2018. Asian longhorned tick, Haemaphysalis longicornis. PennState Extension. https:// extension.psu.edu/asian-longhorned-tick-haemaphysalislongicornis (21 May 2019).

Northeast Regional Center for Excellence in Vector-Borne Diseases (NEVBD). 2018. Longhorned tick a.k.a. cattle tick or bush tick, Haemaphysalis longicornis. http://neregionalvectorcenter.com/longhorned-tick (21 May 2019).

Raghavan RK, Barker SC, Cobos ME, Barker D, Teo EJM, Foley DH, Nakao R, Lawrence K, Heath ACG, Peterson AT. 2019. "Potential spatial distribution of the newly introduced long-horned tick, Haemaphysalis longicornis in North America." Scientific Report. DOI: 10.1038/ s41598-018-37205-2.

Rainey T, Occi JL, Robbins RG, Andrea E. 2018. "Discovery of Haemaphysalis longicornis (Ixodida: Ixodidae) parasitizing a sheep in New Jersey, United States." Journal of Medical Entomology 55: 757-759.

Sleeman J. 2018. Haemaphysalis longicornis detected in the United States. National Wildlife Health Center. https:// prd-wret.s3-us-west-2.amazonaws.com/assets/palladium/ production/s3fs-public/atoms/files/Haemaphysalis $\% 20$ longicornis\%20Detected\%20in\%20the $\% 20$ United $\% 20$ States\%202018.pdf (21 May 2019).
Southeastern Cooperative Wildlife Disease Study (SCWDS). 2019. Surveillance for the exotic tick Haemaphysalis longicornis (longhorned tick) in the eastern United States. https://vet.uga.edu/scwds/H-longicornis (21 May 2019).

Sun J, Liu Q, Lu L, Ding G, Guo J, Fu G, Zhang J, Meng F, Wu H, Song X, Ren D, Li D, Guo Y, Wang J, Li G, Liu J, Lin H. 2008. "Coinfection with four genera of bacteria (Borrelia, Bartonella, Anaplasma, and Ehrlichia) in Haemaphysalis longicornis and Ixodes sinensis ticks from China." Vector-Borne and Zoonotic Diseases 6: 791-796.

Tufts DM, VanAcker MC, Fernandez MP, DeNicola A, Egizi A, Diuk-Wasser MA. 2019. "Distribution, host-seeking phenology, and host and habitat associations of Haemaphysalis longicornis ticks, Staten Island, New York, USA.” Emerging Infectious Diseases 25: 792-796.

Yano Y, Shiraishi S, Uchida TA. 1987. "Effects of temperature on development and growth in the tick, Haemaphysalis longicornis." Experimental and Applied Acarology 3: 73-78. 